Cinémas

Revue d'études cinématographiques

Journal of Film Studies

\title{
« La mémoire, c'est ce qu’il me reste à défaut d'une vue »
}

\section{Bernard Perron}

Volume 5, numéro 1-2, automne 1994

Le Temps au cinéma

URI : https://id.erudit.org/iderudit/1001007ar

DOI : https://doi.org/10.7202/1001007ar

Aller au sommaire du numéro

Éditeur(s)

Cinémas

ISSN

1181-6945 (imprimé)

1705-6500 (numérique)

Découvrir la revue

Citer cet article

Perron, B. (1994). « La mémoire, c’est ce qu'il me reste à défaut d'une vue ». Cinémas, 5(1-2), 91-103. https://doi.org/10.7202/1001007ar

\section{Résumé de l'article}

La temporalité filmique est analysée ici par rapport aux contraintes fixées par le temps de la projection. Parce que la projection d'un film s'effectue en temps réel, la compréhension du récit exige un effort perceptif et cognitif de la part du spectateur. La narration filmique tire profit de cet apport spectatoriel. Analysant Le Silence des agneaux de Jonathan Demme (1990), l'auteur étudie donc les mécanismes de la mémoire, les processus inférentiels ainsi que les deux modes de perception (bottom-up et top-down) qui sont activés lors du visionnement d'un film. 


\title{
«La mémoire, c'est ce qu'il me reste à défaut d'une vue"
}

\section{Bernard Perron}

\begin{abstract}
RÉSUMÉ
La temporalité filmique est analysée ici par rapport aux contraintes fixées par le temps de la projection. Parce que la projection d'un film s'effectue en temps réel, la compréhension du récit exige un effort perceptif et cognitif de la part du spectateur. La narration filmique tire profit de cet apport spectatoriel. Analysant Le Silence des agneaux de Jonathan Demme (1990), l'auteur étudie donc les mécanismes de la mémoire, les processus inférentiels ainsi que les deux modes de perception (bottom-up et top-down) qui sont activés lors du visionnement d'un film.
\end{abstract}

\section{ABSTRACT}

Filmic temporality is here analyzed in terms of the constraints imposed at the time of projection. Because a film is projected in real time, the understanding of its plot requires a perceptual and cognitive effort on the part of the spectator. And filmic narration takes advantage of this spectatorial effort. In an analysis of Jonathan Demme's The Silence of the Lambs (1990), the author studies the memory mechanisms, the inferential processes and the two modes of perception - bottom$u p$ and top-down - that are mobilized in the viewing of a film.

"Scène $A$ " :

Une route, la nuit. Une mention écrite indique : "Memphis, TN". Au volant d'une voiture, une jeune fermme aux cheveux blonds frisés écoute et chante $A n$ 
American Girl de Tom Petty. Dans l'obscurité, un visage portant des lunettes qui permettent de voir dans le noir observe la voiture alors qu'elle se stationne sur le côté d'un bâtiment situé à proximité. La jeune femme sort et salue son chat à la fenêtre. Elle s'avance vers une porte d'entrée et aperçoit un homme seul essayant d'entrer un fauteuil à l'intérieur d'une camionnette. Elle lui propose son aide. (Durée: $1 \mathrm{~min} .50 \mathrm{sec}$.)

Une donnée fondamentale, que nous oublions trop souvent, règle la temporalité filmique. Quelle que soit l'histoire racontée ou quelles que soient les marques temporelles inscrites par la narration, le récit est inévitablement circonscrit par une durée extra-diégétique et extra-filmique, c'est-à-dire le temps de la projection. Au cours d'une séance limitée, standardisée même à 90 ou 120 minutes, le défilement ininterrompu d'informations auditives et visuelles pose quelque difficulté au spectateur. David Bordwell, dont ce texte est grandement tributaire, note que: "Dans un film, l'implacable marche en avant des stimuli demande un effort supplémentaire à la mémoire et aux processus inférentiels du spectateur ${ }^{1} "(1985 a$, p. 33). Pour comprendre le récit, le spectateur devra, comme dans la vie de tous les jours, concentrer ses efforts pour reconstruire mentalement les événements, les actions et les lieux qu'il perçoit. C'est donc par le temps de la perception que les contraintes imposées par le temps de la projection pourront être surmontées. La narration filmique tire avantage de l'activité perceptive et cognitive du spectateur, activité dynamique qui ne correspond pas à un simple enregistrement d'informations. Les données sensorielles doivent être filtrées, transformées, emmagasinées, inférées, comparées avec d'autres données afin que soit bâti un monde cohérent et stable. Par l'entremise du film Le Silence des agneaux (1990) de Jonathan Demme ${ }^{2}$, j'étudierai la tension imposée à la mémoire du spectateur par le temps réel du visionnement ainsi que les mécanismes développés par la narration afin d'en contrôler les effets.

D'abord, il faut spécifier que la mémoire n'est pas faite d'une simple propriété d'emmagasinage d'informations perçues dans un passé éloigné. La mémoire est constructive et doit être consi- 
dérée comme un système de stockage et de traitement d'information. Ce système peut être décomposé en trois opérations successives $^{3}$ :
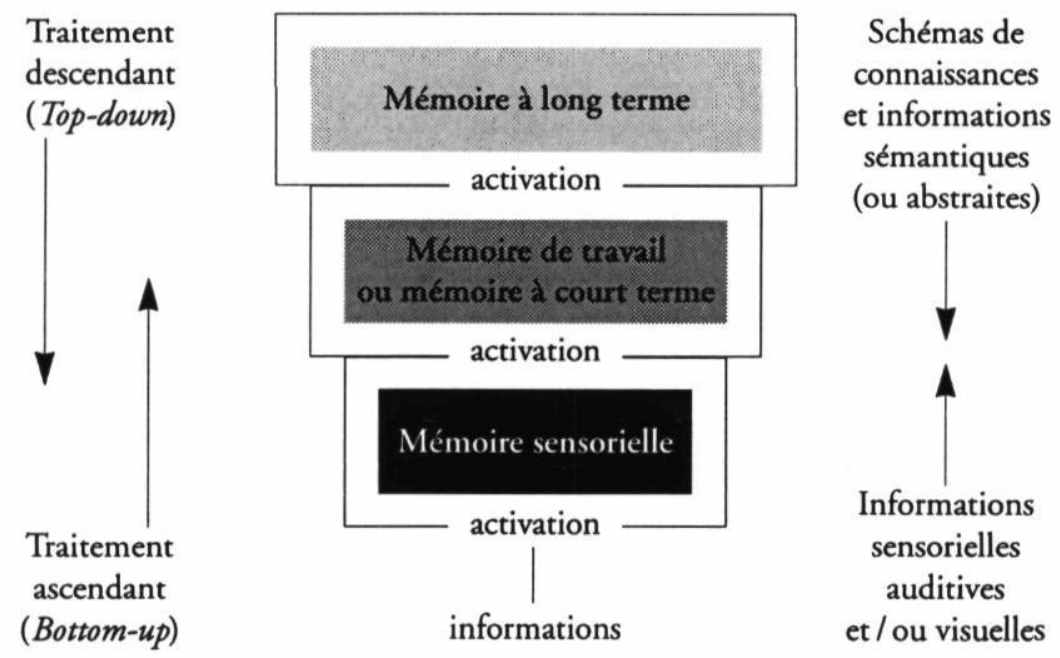

Avant même d'être identifiée et encodée, l'information auditive et / ou visuelle est stockée en mémoire sensorielle. À ce stade-ci, la rétention est très labile (de l'ordre de 300 à 500 $\mathrm{msec}$ ), mais elle permet un transfert vers le second niveau.

On nomme habituellement ce second niveau la mémoire à court terme. Cependant, cette notion a été progressivement abandonnée au profit de celle de "mémoire de travail». Je préfere utiliser ce dernier terme, car il a l'avantage de mettre l'accent sur le traitement de l'information et non pas seulement sur la courte durée de la rétention. De fait, la mémoire de travail est un mécanisme contrôlé et conscient qui agit comme une sorte de processeur central et dirige l'encodage, la rétention et le rappel des informations. Elle possède deux systèmes interreliés (Baddeley) : 1) une tablette visuo-spatiale responsable de la rétention temporaire et de la manipulation d'informations visuelles et spatiales et 2) une boucle articulatoire responsable des informations reliées au langage verbal. La capacité pure de la mémoire de travail est de trois à cinq éléments, que ce soit un objet singulier ou l'idée générale de toute une séquence. Selon le 
temps de présentation et les procédés mnémoniques que l'on utilisera (comme l'apparition successive des éléments à mémoriser ou la révision mentale du spectateur), un plus grand nombre d'éléments pourra être mémorisé. L'augmentation de nombre est fonction d'un déroulement diachronique. En fait, ce second niveau de mémorisation a surtout été étudié avec des contenus verbaux (tels une lettre, un chiffre, un mot ou une phrase). Ainsi, au cinéma, il est certes nécessaire de nuancer cette dite capacité. La polyphonie informationnelle qu'exhibe la "diachronie de synchronie" filmique ne fait pas toujours en sorte de concentrer l'attention spectatorielle sur un élément particulier (entre autres par l'entremise d'un gros plan). Le spectateur doit traiter l'image (et le son) afin d'en extraire les éléments à encoder. La notion de connaissance préattentive pourrait permettre d'expliquer la facilité avec laquelle le spectateur comprend ou perçoit l'épaisseur de signes du cinéma. Cette connaissance permet au spectateur d'inférer des informations sur la représentation attendue et de concevoir une carte mentale des éléments importants. L'information stockée en mémoire de travail ne se dégrade pas avant quelques secondes et même, dans le cas d'une révision mentale, peut être conservée durant quelques dizaines de secondes.

Il faut plusieurs secondes pour stocker une information dans la mémoire à long terme, car celle-ci conserve des informations abstraites. En d'autres mots, les informations sensorielles sont dépouillées de leur attribut de surface pour être traitées, encodées et organisées à partir de leur sens. Plus la période de temps écoulée entre l'encodage et le rappel est longue, plus l'information peut devenir abstraite jusqu'à disparaître totalement (nous oublions, par exemple, la fin d'un film vu il y a plusieurs mois). Multimodulaire, la mémoire à long terme est principalement une mémoire sémantique dont l'utilisation quotidienne est, par endroits, inconsciente. En fait, lorsqu'il s'agit d'envisager notre connaissance des faits, des choses et des êtres, la mémoire à long terme se scinde en deux systèmes: la mémoire sémantique et la mémoire épisodique. Alors que la première contient les informations nécessaires à l'utilisation du langage, la seconde est autobiographique et contient les souvenirs d'événements et 
d'expériences personnels. C'est à ce niveau que l'on retrouve certains schémas de connaissance. Cette notion a été introduite en 1932 par Frédéric C. Bartlett qui définit le schéma comme "[...] une organisation active de réactions passées, ou d'expériences passées, qui doivent toujours être supposées intervenir dans une quelconque réponse organique bien adaptée " (Bartlett, p. 201). La mémoire à long terme représente notre connaissance préalable et de la vie de tous les jours et des structures narratives. Elle contient une série de scripts (c'est-à-dire une organisation temporelle définie et typique d'événements et d'actions comme, par exemple, le script restaurant), de scènes (soit une organisation définie et typique de relations spatiales ou physiques d'objets dans un lieu comme, par exemple, la scène cuisine) et d'expectatives qui nous aide à comprendre et à traiter de nouvelles informations. Cependant, suivant les souvenirs emmagasinés en mémoire épisodique, chaque individu possède également des scripts et des scènes qui lui sont personnels et qui peuvent s'écarter des structures usuelles.

En définitive, la mémoire sert de connecteur, de filtre et de guide à notre perception. Pour cette raison, elle conditionne le mode de perception utilisé par le spectateur pour traiter les informations auditives et/ou visuelles qui lui sont présentées. Les études en sciences cognitives distinguent deux modes de perception ou de traitement d'information. Ascendant, le premier mode est dirigé par les données (bottom-up), c'est-à-dire qu'il repose sur les informations sensorielles auditives et / ou visuelles fournies ponctuellement dans le film. Descendant, ce second mode est orienté vers les concepts (top-down), c'est-àdire qu'il s'appuie sur les schémas de connaissance du spectateur. Nous allons voir de quelle manière ceux-ci fonctionnent à travers l'activation de la mémoire.

$\mathrm{Si}$ je vous demandais, en prenant en considération l'importante quantité d'informations visuelles et auditives qu'elle pouvait comporter, de me décrire en détail la "scène $A$ " extraite du Silence des agneaux, scène qui introduit cet article... Qu'est-ce que, par exemple, chantait la jeune femme? De quelle couleur était la voiture? Combien y avait-il de fenêtres au mur faisant face au stationnement? De quel côté était le chat? Etc. De toute 
évidence, il vous serait sensiblement difficile de le faire sans omettre quelques détails. Vous ne pouviez pas prévoir que je vous poserais une telle question. Vos expectatives ou attentes vous ont certainement poussé à vous représenter la scène et à la comprendre, c'est-à-dire à construire une représentation mentale cohérente. Cependant, ne sachant pas que toutes les petites particularités visuelles et auditives seraient ultérieurement considérées et ayant dû porter jusqu'à maintenant votre attention sur la lecture du présent texte, j'imagine que peu d'entre vous avez révisé mentalement la scène de façon répétitive afin de ne rien oublier. Parce qu'un certain laps de temps réel s'est écoulé et parce qu'il vous serait impossible de retourner en arrière "audiovoir" (suivant l'expression de Michel Chion) la scène du film ${ }^{5}$, vous devez vous contenter des images et des sons que vous avez conservés en mémoire et que vous pouvez, en ce moment, vous rappeler.

"Une performance [de mémorisation] est limitée par la demande qu'exerce une tâche sur le système cognitif ${ }^{6}$ " (Ellis et Hunt, p. 52). Parce que la quantité d'informations sensorielles est très grande et parce qu'il n'est pas en mesure de juger en tout temps de ce qui est important, le spectateur ne peut pas retenir ou mémoriser tous les détails, toutes les informations de surface d'une scène. Celles-ci se transforment continuellement. Une nouvelle information de surface en supprime une ancienne dans la mémoire de travail. La capacité de rétention étant de quelques secondes, le spectateur doit encoder le sens général d'une scène en mémoire à long terme afin de pouvoir traiter la suite du défilement. Le spectateur doit traiter les informations de façon ascendante (bottom-up). Il doit obligatoirement partir des données fournies par le film afin de poser des hypothèses et élaborer petit à petit une représentation abstraite. Ainsi, pour s'assurer que le spectateur retienne les informations les plus importantes, la narration doit les signaler.

Parce que le temps de visionnement pose des contraintes à la mémoire, l'habileté du spectateur à construire une fabula cohérente dépend de références répétées aux événements de l'histoire. Afin de garder les grandes lignes de l'action claires et afin d'assurer que les hypo- 
thèses appropriées soient émises, la narration doit réitérer les plus importantes coordonnées causales, temporelles et spatiales de la fabula. La répétition peut intensifier la curiosité et le suspense, ouvrir et fermer des brèches, diriger le spectateur vers les hypothèses les plus probables ou vers les moins vraisemblables, retarder la révélation de certaines issues, et assurer que la quantité de nouvelles informations au sujet de la fabula ne soit pas trop grande ${ }^{7}$ (Bordwell, 1985a, p. 80).

Le Silence des agneaux exemplifie parfaitement le jeu et la portée de la répétition des éléments dramatiques importants. L’une des intrigues tourne autour de l'évasion du $\mathrm{D}^{r}$ Hannibal Lecter (Anthony Hopkins) grâce à l'agrafe d'un stylo à bille dérobée au docteur en chef. Cette évasion commence à la $74^{\circ}$ minute du film alors que le $\mathrm{D}^{r}$ Lecter régurgite ladite agrafe. Au préalable, pour bien marquer la cohérence de cette action, le réalisateur a, par la narration et de diverses façons, tenu à signaler l'importance de l'objet :

1) $8^{\prime} \min =L e D^{\prime}$ Chilton (Anthony Heald) tient son stylo à bille dans ses mains lorsqu'il discute avec l'agent Clarice Starling (Jodie Foster) lors de la toute première visite de cette dernière (plans moyen et rapproché).

2) $9^{\mathrm{c}} \min =$ En route vers la cellule du $D^{r}$ Lecter, le $D^{\prime}$ Chilton explique à Starling: "Ne lui remettez que des feuilles de papier. Surtout pas de stylo ou de crayon ni agrafe, ni trombone sur le papier. S'il tente de vous transmettre quoi que ce soit, ne l'acceptez pas."

3) fin $56^{\circ} \min =$ Gros plan du profil du D Chilton. Il écoute à l'aide d'un magnétophone une conversation entre Starling et le D Lecter. Il porte sa main à son oreille et exhibe ainsi son stylo à bille bien en vue.

4) fin $58^{\prime}$ min $=$ Le D Chilton discute avec le D Lecter dans sa cellule. Il est couché sur le lit et joue avec son stylo à bille.

5) $59^{\circ} \min =$ "mémorisation du stylo à bille "

Plan 1: Panoramique sur le stylo à bille en plan rapproché $(5,5 \mathrm{~s})$

Plan 2: Lent zoom in en gros plan sur le regard du

$\mathrm{D}^{\prime}$ Lecter $(4,5 \mathrm{~s})$. 
Plan 3: Lent zoom in en gros plan sur le stylo (4s).

Plan 4 : Lent zoom in en gros plan sur le regard du $\mathrm{D}^{\prime}$ Lecter $(5,5 \mathrm{~s})$.

Plan 5: Lent zoom in en très gros plans sur le stylo (4s).

Plan 6: Lent zoom in en gros plan sur le regard du $\mathrm{D}^{\prime}$ Lecter $(4,5 \mathrm{~s})$.

6) $61^{\mathrm{e}} \mathrm{min}=\AA \grave{A}$ l'aéroport de Memphis, le D' Chilton ne trouve pas son stylo pour signer le formulaire de transfert du $\mathrm{D}^{\prime}$ Lecter. Plan rapproché de ses mains qui fouillent dans son veston. Un policier lui donne le sien.

7) $74^{\circ} \mathrm{min}=\mathrm{Le} \mathrm{D}^{r}$ Lecter régurgite l'agrafe de stylo à bille afin de s'évader.

À la $9^{\mathrm{c}}$ minute du film, une réplique du $\mathrm{D}^{\mathrm{r}}$ Chilton révèle déjà qu'il est dangereux de fournir au $\mathrm{D}^{r}$ Lecter des petits objets en métal. Cependant, cette information verbale est transmise très rapidement et dans un contexte qui ne prête pas à un encodage spécifique en mémoire à long terme. De la même façon, ne connaissant pas la valeur narrative et sémantique du stylo du $\mathrm{D}^{\prime}$ Chilton, le spectateur ne lui accorde aucune signification lorsque celui-ci apparaît avant (à la $8^{\mathrm{c}}$ minute) et après (aux $56^{\circ}$ et $58^{c}$ minute) la directive du $\mathrm{D}^{r}$ Chilton. Le stylo est une information de surface dont la mémorisation est labile. Ce n'est qu'à la $59^{c}$ minute, alors que la réplique du début est oubliée ${ }^{8}$, que l'objet va prendre un sens particulier. Pendant 28 secondes, un montage entre le regard du $\mathrm{D}^{r}$ Lecter et le fameux stylo incite (ou oblige) le spectateur à saisir toute l'importance, sur le plan de l'action, de l'objet et à l'encoder sémantiquement en mémoire à long terme. Pour mieux saisir l'importance du stylo, le zoom in en très gros plan l'isole du décor, de sorte que l'information glisse pour ainsi dire d'emblée à un niveau différent. Cette scène met en évidence l'importance à accorder au rythme de présentation de l'information narrative. Si la vitesse de présentation est trop rapide, la mémoire de travail n'a pas le temps d'effectuer un traitement profond de l'information et de la soumettre à la mémoire à long terme. Dans le cas présent, le spectateur bénéficie d'un temps suffisamment long pour traiter cette 
information de façon plus abstraite. La narration favorise le mode de perception de type ascendant (bottom-up) en fournissant au spectateur des données explicites afin qu'il puisse créer certaines hypothèses conceptuelles. Lorsque le $\mathrm{D}^{\prime}$ Chilton cherche son stylo une minute plus tard, la répétition augmente le suspense. Puisque la narration hollywoodienne appelle des hypothèses à la fois très probables et très exclusives, elle limite le travail perceptif et cognitif du spectateur à un nombre restreint d'inférences (Bordwell, 1985b, p. 24-41).

Maintenant, si je vous demandais de prédire la fin de la "scène $A$ ". Qu'allait-il se produire? La jeune femme allait-elle porter secours à l'homme et entrer chez elle? Allait-elle se faire agresser? De toute évidence, même hors contexte' ${ }^{9}$, l'agression qui se produit dans la "scène $A$ " est très prévisible et pour deux raisons. La première est que la narration hollywoodienne raconte des histoires canoniques obéissant à une structure causale stricte. Nous venons de le mentionner, les hypothèses possibles sont extrêmement restreintes. La seconde raison qui explique cette capacité d'inférences repose sur le mode de perception de type descendant (top-down). Cette fois-ci, ce sont les concepts et les schémas de connaissance du spectateur qui servent de principe organisateur. D'entrée de jeu, notre connaissance des structures narratives oriente le récit vers une suite d'actions particulières en nous permettant de postuler sur la suite du récit. Conséquemment, l'instance spectatorielle retourne au film afin d'y trouver des informations qui vont corroborer, au fur et à mesure du déroulement de l'action, l'organisation d'accueil qu'il s'en est faite. Le rappel, en mémoire de travail, de ces schémas, scripts ou scènes, rend immédiatement disponible un nombre considérable d'informations. Ceux-ci ne surchargent pas l'espace de traitement puisqu'ils constituent des blocs abstraits, c'est-àdire qu'ils représentent plutôt un seul élément et non plusieurs.

Dans Le Silence des agneaux, le mode de perception descendant (top-down) du spectateur est particulièrement sollicité lors de l'ultime offensive policière menée contre la (supposée) maison du tueur en série ${ }^{10}$. Cependant, dans le cas présent, la narration falsifie - ou pervertit - une règle discursive du cinéma classique. On le sait, le montage alterné se définit comme un 
montage qui alterne entre deux ou plusieurs lignes d'actions se déroulant simultanément dans des lieux séparés par une certaine distance diégétique. La séquence du Silence des agneaux montre d'abord la maison de l'extérieur afin d'établir le premier lieu de l'alternance. Dès le second plan, en montrant une image du tueur dans sa cave, la narration fixe implicitement une relation "extérieur/ intérieur». N'ayant d'ailleurs jamais vu l'extérieur de la vraie maison du tueur (un panoramique le lui montrera à la fin du suspense), le spectateur récupère donc en mémoire de travail ce schéma de connaissance filmique relié au montage alterné pour prédire l'action du film. De la sorte, il obéit sans doute à la "persistance d'une attitude" (persistence of set) qui veut qu'une fois qu'une stratégie ou une règle permettant de résoudre un problème est apprise, nous avons tendance à l'appliquer même si la situation change (Ellis et Hunt, p. 226-228). La discontinuité sonore, de même que les deux raccords qui lient les plans du faux fleuriste sonnant à la porte et ceux de la sonnerie dans la cave, confirment a priori la représentation mentale de l'alternance "extérieur/intérieur". Ce n'est qu'au moment où le tueur ouvre la porte à l'agent Starling que l'hypothèse du spectateur est réfutée. $\mathrm{La}$ "réussite» de la séquence s'appuie donc sur le processus inférentiel du spectateur.

Cependant, la perversion narrative du Silence des agneaux va beaucoup plus loin. Dès l'ouverture, une mention écrite apparaît au bas de l'écran à droite. Ce même procédé est employé tout au long des 96 minutes suivantes:

1) ouverture du film = Woods near Quantico, VA.

2) $31^{\mathrm{c}} \mathrm{min}=$ Memphis, $T N$.

3) $35^{\circ} \mathrm{min}=$ Clay Country, WVA.

4) $60^{\circ} \mathrm{min}=$ Memphis International Airport.

5) $64^{\circ} \mathrm{min}=$ Shelby Country House.

6) $86^{\circ} \mathrm{min}=$ Belvedere, Ohio.

7) $95^{\circ} \mathrm{min}=$ Air National Guard HQ, Chicago, IL.

8) $96^{\mathrm{c}} \mathrm{min}=$ Calumet City, IL.

Ces mentions écrites désignent l'endroit où se déroule l'action de manière plus ou moins précise, soit par le nom d'une ville et d'un État (en 1, 2, 3 et 6), soit par la dénomination d'un lieu 
précis (en 4, 5 et 7). Toutefois, l'apparition de ces indications spatiales est sporadique. Chaque séquence et chaque lieu ne sont pas caractérisés de cette façon. Un grand nombre de transitions entre les scènes se réalise sans indication écrite. Par conséquent, le spectateur est amené à leur conférer une certaine autorité. En réalité, ces indications ont un effet de primauté (Ellis et Hunt, p. 73 et Bordwell, 1985a, p. 38). Elles servent de cadres de référence, comme un titre sert à identifier et à subordonner une série d'actions afin d'en favoriser la compréhension. Par leur présence, elles engagent un traitement ascendant (bottom-up). Le spectateur va construire la représentation d'un lieu en fonction de l'indication fournie. Mais d'autre part, la répétition préalable de cette stratégie et le rappel d'une scène qui en découle mobilisent également une représentation conceptuelle renvoyant à un mode de traitement descendant (top-down). Le spectateur utilise un schéma de connaissance relié au lieu. On comprendra donc que les deux modes de perception sont tous deux appelés lors du traitement d'une information et qu'ils opèrent en interaction continuelle. C'est d'ailleurs pour cette raison que dans le schéma présenté en début d'article, les flèches de gauche qui représentent les deux modes de perception se chevauchent et se croisent en mémoire de travail. Dès lors, lorsque l'indication "Calumet City, IL " apparaît au bas du plan d'ensemble de la maison (un establishing shot), le spectateur ne peut que suivre la logique intrinsèque du film et désigner cet endroit comme étant le lieu de l'action. Comment peut-il savoir que la maison du tueur est à Belvedere, Ohio? Comment peut-il savoir que la règle des indications spatiales que lui a fait mémoriser la narration est tout à coup rejetée? Comme le note avec justesse Bordwell: "Chaque film forme son propre spectateur " "(1985a, p. 45).

Ludique et pervers, Le Silence des agneaux nous fait prendre conscience que "[...] l'activité perceptive et cognitive [du spectateur] va toujours au-delà de l'information qui lui est donnée ${ }^{12}$ " (Bordwell, 1989, p. 18) et que la narration filmique en tire profit. Au cours du temps réel de projection, deux modes de perception interviennent ensemble au cours du visionnement d'un film: l'un est ascendant (bottom-up) et l'autre est descendant (top-down). 
Enfin, j'aimerais conclure en me référant à une autre scène du film de Jonathan Demme. À la vue d'une esquisse détaillée de Florence en Italie sur le mur gauche de la cellule du $\mathrm{D}^{r}$ Lecter, l'agent Starling lui demande s'il est capable d'autant de détails uniquement de mémoire. À cette question, le $\mathrm{D}^{r}$ Lecter répond l'intitulé de mon intervention: "La mémoire, c'est ce qu'il me reste à défaut d'une vue. " Le spectateur de cinéma peut paraphraser cette réplique: "La mémoire, c'est ce qu'il me reste à défaut d'une libre audio-vision au cours du visionnement d'un film." Espérons donc que ce spectateur se souvienne du stratagème du Silence des agneaux.

\section{Université de Montréal}

\section{NOTES}

1 Ma traduction.

2 Rappelons qu'en 1991, ce film gagna entre autres l'Oscar du meilleur film et l'Oscar du meilleur réalisateur.

3 Orienté en fonction du présent exposé, ce schéma ne représente certes pas les mécanismes de la mémoire dans toute leur complexité. Pour un exposé plus précis de ces mécanismes et des niveaux de la mémoire, voir entre autres de J.-F. Richard, Les Activités mentales. Comprendre, raisonner, trouver des solutions (Paris: Armand Colin, 1990) ou Claudette Fortin et Robert Rousseau, Psychologie cognitive. Une approche de traitement de l'information (Québec: Télé-Université, 1993).

4 Une traduction du texte original anglais par Michel Fayol dans Le Récit et sa construction. Approche de psychologie cognitive (Neuchâtel/ Paris: Delachaux/Niestlé, 1985, p. 38).

5 On pourrait ici s'objecter en disant que le magnétoscope permet maintenant ce retour en arrière. Cependant, si ce n'est que pour écouter à nouveau une réplique immédiate mal entendue, l'opérateur de magnétoscope se plie tout de même à l'implacable marche en avant du temps filmique.

6 Ma traduction.

7 Ma traduction.

8 D'une certaine perspective, cette information ne doit-elle pas être oubliée afin de donner plus de cohérence à cette intrigue? En effet, comment se fait-il que le $\mathrm{D}^{\prime}$ Chilton, qui a pris soin de prévenir l'agent Starling d'une règle stricte à la $9^{c}$ minute, oublie son stylo à bille dans la cellule du $\mathrm{D}^{\prime}$ Lecter? La scène se termine avant sa sortie et laisse ainsi une brèche narrative qui ne sera jamais fermée.

9 Dans le film, la "scène $\mathrm{A}$ " est précédée d'une réplique du $\mathrm{D}^{t}$ Lecter : "Buffalo Bill doit certainement chercher sa prochaine fiancée."

10 Cette séquence, qui débute à la $96^{\mathrm{C}}$ minute, alterne entre deux espaces: 1) l'extérieur de la maison envahi par les policiers et 2) l'intérieur de la cave où le tueur détient la victime (de la "scène $A$ ").

11 "Every film trains its spectator."

12 Ma traduction. 


\section{OUVRAGES CITÉS}

Baddeley, Alan. "The Uses of Working Memory", dans P. R. Solomon (dir.), Memory : Interdisciplinary Approaches. New York: Springer-Verlag, 1989, p. 107-123.

Bartlett Frederic C. Remembering a Study in Experimental and Social Psychology. Londres: Cambridge University Press, 1964.

Bordwell, David. "A Case for Cognitivism", Iris, $\mathrm{n}^{\circ} 9$ (automne 1989).

Bordwell, David. Narration in the Fiction Film. Madison: The University of Wisconsin Press, 1985a.

Bordwell, David. "Classical Narration", The Classical Hollywood Cinema. Film Style o Mode of Production to 1960 (collectif). New York: Columbia University Press, 1985 b.

Ellis, Henry C. et Hunt, R. Reed. Fundamentals of Human Memory and Cognition. Iowa : Wm C. Brown Publishers, 1989.

Fayol, Michel. Le Récit et sa construction. Une approche de psychologie cognitive. Neuchâtel / Paris : Delachaux / Niestlé, 1985.

Fortin, Claudette et Rousseau, Robert. Psychologie cognitive. Une approche de traitement de l'information. Québec: Télé-Université, 1993.

Lieury, Alain. La Mémoire. Du cerveau à l'école. Paris: Flammarion, 1993.

Norman, Donald A. et Rumelhart, David E. "Memory and Knowledge ", dans D. Norman et D.E. Rumelhart (dir.), Explorations in Cognition. San Francisco: W. H. Freeman and Company, 1975, p. 3-32.

Richard, Jean-François. Les Activités mentales. Comprendre, raisonner, trouver des solutions. Paris: Armand Colin, 1990.

Simon, Jean-Paul. «Remarques sur la temporalité cinématographique dans les films diégétiques", dans D. Chateau, A. Gardies et F. Jost, Cinémas de la modernité: Fïlms, théories. Paris: Klincksieck, 1981, p. 57-73.

Tannen, Deborah. "What's in a Frame? Surface Evidence for Underlying Expectations", dans Roy O. Freedle (dir.), New Directions in Discourse Processing. Volume II: Advances in Discourse Processes. Norwood: Ablex Publishing Corporation, 1979, p. $137-181$. 